\title{
Studying Nuclei with High-Intensity Electron Beams
}

\begin{abstract}
Bright perspectives for the study of polarization phenomena in complex hadronic systems are in sight. This follows preliminary results from an internaltarget scattering experiment investigating the quadrupole shape of polarized deuterium using high-intensity electron beams at NIKHEF's $800 \mathrm{MeV}$ pulse stretcher and storage ring AmPS, which started operating in 1992.
\end{abstract}

Deuteron - the simplest atomic nucleus after hydrogen - is essentially spherical in shape with an angular momentum quantum number $I=0$. Much is known about this $I=0$ component thanks to experiments, among others, carried out with the linear accelerator MEA at the Dutch National Institute for Nuclear Physics and High-Energy Physics (NIKHEF) in Amsterdam. This is a joint venture of the Dutch funding agency FOM and Dutch universities, where intense bunches of electrons with a small wavelength, corresponding to a resolution of about $5 \%$ of the dimensions of the system, are scattered off unpolarized deuterium nuclei. The spherically symmetric part is a direct consequence of the central component of the strong interaction between the proton and the neutron ("central" means directed along the line connecting the centres-of-mass of the proton and the neutron, and independent of the particles' relative velocity vectors and spin orientation).

An experimentally unknown shape element (since its magnitude is small and difficult to measure) has the form of a $I=2$ quadrupole (Fig. 1). It is related to the so-called tensor interaction between the proton and the neutron; the tensor force is poorly known, has a short range and is of fundamental importance. When the $I=2$ shape rotates in space, the average shape observed cannot be distinguished from the spherically symmetric $I=0$ shape.
The strategy needed to measure the quadrupole component is clear: the deuterium $\left({ }^{2} \mathrm{H}\right)$ nuclei must be polarized when probing the shape using scattered electrons. One way to fix the orientation of ${ }^{2} \mathrm{H}$ is to use solid deuterated ammonia $\left(\mathrm{ND}_{3}\right)$ at $2 \mathrm{~K}$ in a container placed in a strong magnetic field. This "frozen-spin" method has several disadvantages: the electrons also scatter off the container walls and the nitrogen atoms, the electron paths are perturbed by the strong mag-

\section{AmPS}

AmPS started operating in 1992 and began delivering beams for physics in the spring of 1993. Major goals were reached in 1993, including a $500 \mathrm{Mev}$ extracted beam of $3 \mu \mathrm{A}$ at a duty factor of up to $50 \%$ in the stretcher mode, and stable operation with approaching 100 $\mathrm{mA}$ of circulating current with a lifetime up to 30 minutes in a storage-stacking mode involving the injection and stacking of $0.7 \mu \mathrm{s} / 30 \mathrm{~mA}$ current pulses. The principle of using AmPS as a synchrotron to raise the energy of the circulating beam has been successfully demonstrated; polarized electrons will be stored in mid-1995. The next deadline for proposals for beam time is 26 August 1994.
Fig. 2 - The AmPS polarized deuterium internal-target facility (a collaboration between NIKHEF, ETH-Zürich, the University of Wisconsin, and Arizona State University). An atomic beam source generates a flux of deuterium molecules which is separated into atoms using an intense high-frequency field. Electrons bound to the deuterium nuclei are then aligned by means of Stern-Gerlach separation (focussing of hyperfine states in a tapered sextupole electromagnet with a $1.1 \mathrm{~T}$ tipfield). The resulting flux of 2 $\times 10^{16}$ polarized deuterium nuclei per second are injected into a $T$-shaped storage cell with a $400 \mathrm{~mm}$ long by 20 $\mathrm{mm}$ in diameter section traversed by a circulating electron beam. A 100 times increase in the effective density of polarization is achieved provided polarization is kept intact in spite of each ${ }^{2} \mathrm{H}$ atom encountering about 500 collisions with the cell walls before leaving the storage cell. Using specially prepared Teflon or similar coatings and an optimum cell temperature it is possible to maintain the initial (injected) degree of polarization to above $90 \%$. The effective target density achieved so far is $3 \times 10^{13}$ atoms $/ \mathrm{cm}^{2}$, a value that will be improved by a factor of 5 in the near future by cooling the walls and by reducing the diameters of entrance and exit tubes of the storage cell. The walls of the storage cell (with a typical thickness of about $100 \mu \mathrm{m}$ ), are approximately $10^{8}-10^{9}$-times heavier than the ${ }^{2} \mathrm{H}$ target, so the halo of the electron beam that can interact with the cell walls must contain at least 109-1010-times less electrons than the central electron beam. This has been achieved using detailed Monte Carlo simulations combined with beam stabilization.
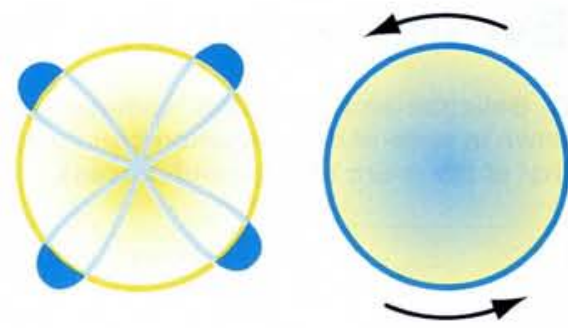

Fig. 1 - An schematic illustration of the $I=2$ quadrupole component of the shape of the deuteron nucleus.

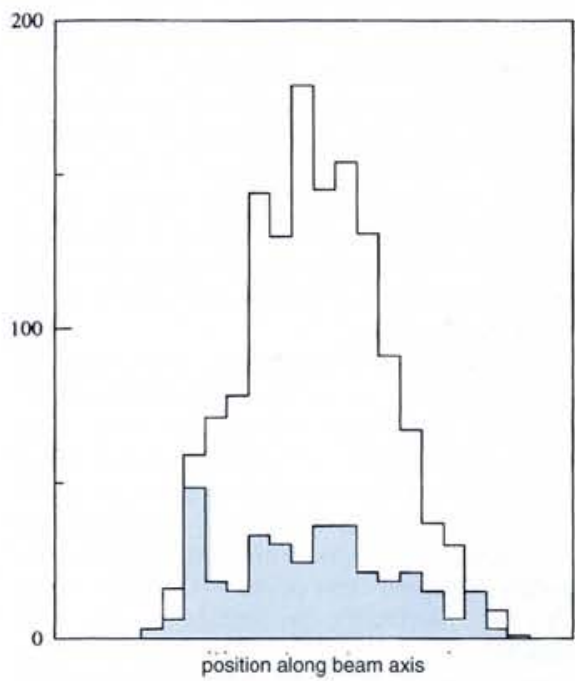

Fig. 3 - The measured scattering rate as a function of the position along the beam axis with (unshaded histogram) and without ${ }^{2} \mathrm{H}$ filling of the storage cell demonstrates the excellent signal-to-background ratio.

netic field, and even a small electron current $(<0.1 \mu \mathrm{A})$ destroys the polarization. These factors make the measurement of the small $I=2$ effect at best marginal. An interesting new method is to inject a high-intensity beam of polarized deuterium atoms into a T-shaped storage cell with open ends through which the electron beam can pass undisturbed (Fig. 2). Compared to $\mathrm{ND}_{3}$, the target will be ultrathin. Moreover, the cross-section for electrondeuteron collisions is small. A high-intensity electron beam of at least $100 \mathrm{~mA}$ is therefore required to achieve a useful scattering rate. The solution is in principle both simple and effective: use the electrons stored in a circulating beam about $10^{9}$ times for the scattering process.

At the AmPS internal gas-target experiment, electrons are detected at a central angle of $35^{\circ}$ in coincidence with the ejected protons by means of an electromagnetic calorimeter comprising $60 \mathrm{Csl}$ scintillator crystals. The position of each event is recorded using a range telescope (illustrated in Fig. 2) made from 16 layers of plastic scintillator material to detect the protons. Fig. 3 shows the measured scattering rate as a function of the position along the beam axis with and without ${ }^{2} \mathrm{H}$ filling of the storage cell. The signal-to-background ratio is particularly good, being more than a factor of 10 better than elsewhere; it has allowed a first measurement of the $I=2$ component.

P.K.A. de Witt Huberts J.F.J. van den Brand NIKHEF, Amsterdam 